\title{
SO(10) SUSY GUTs from M theory
}

\section{Crispim Romao*}

School of Physics and Astronomy, University of Southampton, Southampton, SO17 1BJ, UK

E-mail: m.crispim-romaodsoton.ac.uk

\begin{abstract}
$M$ Theory compactified on $G_{2}$ manifolds provides a rich framework for model building and phenomenology. In this communication we present the latest developments of $M$ Theory on $G_{2}$ manifolds with $S O(10)$ as the Grand Unification Theory group, by making use of the discrete symmetries of the internal manifold. We show that we are generically led to a new solution to the doublet-triplet problem, which requires the presence of an extra $\overline{\mathbf{1 6}}_{X}, \mathbf{1 6}_{X}$ vector-like family at the $\mathrm{TeV}$ scale. Since the unification group is broken by discrete Wilson lines, the surviving gauge group has an extra $U(1)$, that once broken can generate neutrino masses in a type-I seesaw mechanism. The main prediction of the framework is the existence of light states with the quantum numbers of a $\overline{\mathbf{1 6}}_{X}, \mathbf{1 6}_{X}$ vector-like family, which could show up in future LHC searches.
\end{abstract}

18th International Conference From the Planck Scale to the Electroweak Scale 25-29 May 2015

Ioannina, Greece

* Speaker. 


\section{Introduction and motivation}

The recent discovery of a Standard Model (SM) like Higggs [1, 2, 3] is stressing the need to understand the Electroweak Scale (EWS), namely its stability and the Higgs mass. Ultimately one has to answer to the question, why is the EWS 16 orders of magnitude below the Planck scale? This is the so-called hierarchy problem.

A vast framework used to address these issues it the one of Supersymmetry (SUSY), where the mass parameters of scalars are stabilised by being protected against quantum corrections that are sensitive to the quartic value of the scales to which the scalars couple. While this is true for global SUSY models, one needs to go beyond to its local form, Supergravity (SUGRA), if one wants an extended framework with natural low scale mass generating mechanisms, depending on the ultra-violet (UV) completion of the theory.

The search for SUSY partners have been so far negative[4, 5, 6], pushing the bounds of their masses above $\mathscr{O}(1 \mathrm{TeV})$ while naturalness suggests they should be just above the EWS. However, naturalness has led hitherto supersymmetric model building to take place mostly in the context of the Minimal Supersymmetric Standard Model (MSSM) or conservative variations of it, usually exploring special parts of the parameter space. As such, the experimental bounds mostly constrain the MSSM, while other, more general and complete, models remain relatively untested.

It is then necessary to study models beyond the MSSM, their limits and predictions. In doing so one is presented with the fact that are virtually infinite possibilities to extend the MSSM, and one has to ask how and where to do we extend this study? Fortunately there is a rich guideline for this enquire, String pheory phenomenology.

Nowadays we understand the different string theories to be related by an intricate web of dualities, and eventually all of them to be different limits of an underlying 11 dimensional theory, the so-called $M$ theory. A definite microscopic formulation of $M$ theory is yet to be developed, but its low-energy limit, an unique 11 dimensional SUGRA, has been the window to many advancements and insights. Consequently we turn to the study of the phenomenology of this 11 dimensional supergravity upon suitable compactification of the extra seven dimensions. Results over the past two decades have shown that the combination of spontaneously broken SUSY and moduli stabilisation in $M$ theory can be a very useful guideline for model building, as it has been explored $[7,8,9,10,11]$. The framework has been shown to lead to effective global supersymmetric models with distinctive features and few parameters at the GUT scale.

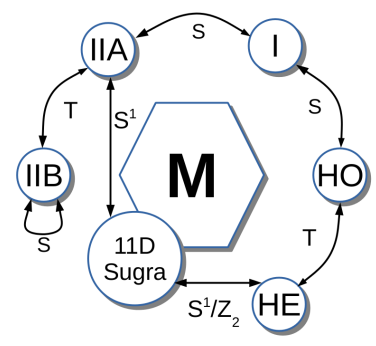

Figure 1: $M$ Theory and String Theory web of dualities

As we will explain in this communication, $M$ theory compactified on a manifold of $G_{2}$ holonomy and without fluxes leads elegantly to four dimensional models with $\mathscr{N}=1$ supersymmetry, 
grand unified theories (GUTs) based on simple groups, hierarchically small mass parameters generation, and natural discrete symmetries of the internal manifold that provide a solution to the doublet-triplet splitting problem.

It's the purpose of this work to extend the scope of the $M$ theory approach from the previously considered $S U(5) / \mathrm{MSSM}$ case arising from $M$ theory on $G_{2}$ manifolds - the so-called $G_{2}$ MSSM [8] - to $S O(10)$ [12]. As such, entire fermion family $Q, u^{c}, d^{c}, L, e^{c}, N$, including a charge conjugated right-handed neutrino $N$, is unified within a single $\mathbf{1 6}^{m}$ representation. In particular we focus on the Higgs doublet-triplet splitting problem and present a solution, which turns out to be necessarily quite different from the $G_{2}$-MSSM, since the customary solution of making the coloured triplets heavy $[13,14,15]$ fails. As a consequence, our solution leads to distinct phenomenological constraints and predictions.

In the remainder of this work, we start by reviewing some basic ideas and results from $M$ theory compactified on $G_{2}$ manifolds without fluxes. This will introduce the main features of $M$ theory model building with the phenomenological features being explicitly discussed. Afterwards we embark on a discussion of the new $S O(10)$ case and how we are led to additional light states at the reach of the LHC.

\section{2. $M$ Theory model building}

When compactifying $M$ Theory on $G_{2}$ manifolds, both Yang-Mills fields and chiral fermions arise from very particular kind of singularities in the extra dimensions [16, 17]. Yang-Mills fields are supported on three-dimensional subspaces of the seven extra dimensions along which there is an orbifold singularity. Chiral fermions arise from additional localised points at which there is an $A, D, E$-type conical singularity, and hence couple to the Yang-Mills fields. Therefore, different GUT multiplets are localised at different points in the extra dimensions.

Compact manifolds of $G_{2}$ holonomy - being Ricci flat and having a finite fundamental group - do not admit continuous symmetries, but can have with discrete symmetries. If present, they play a very important role in the physics. In particular, for the $S U(5)$ case, Witten showed that such symmetries can solve the doublet-triplet splitting problem [18].

The compactified space plays a crucial role on setting the effective 4-dimensional field theory. For example, if the compactified space has a non-trivial fundamental group, i.e. when it has holes or handles, the GUT group can be broken by Wilson lines. A consequence of having non-trivial fundamental group is that the compactified space has non-contractible closed loops. Consequently there will be non-vanishing gauge field configurations such that, along a non-contractible loop, we have

$$
\mathscr{W}=\mathscr{P} \exp \oint A_{k} d^{k} \neq 1,
$$

where $A$ is the GUT gauge field, $\mathscr{P}$ represents path-ordering, and $k=5, \ldots, 11$ runs through the internal space dimensions.

The above quantities are not local and cannot be gauged away, and are therefore observables. To see this we notice that consider a singularity in the extra dimensions supporting a GUT irrep $\Psi$, localised along a Wilson line path, and let it absorb the respective quantities $\mathscr{W}$

$$
\Psi \rightarrow \Psi^{w}=(\mathscr{W} \Psi)
$$


the surviving gauge group will now be composed of the elements of the GUT group that can commute with $\mathscr{W}$

$$
\Psi^{w} \rightarrow(\mathscr{W} g \Psi)=g \Psi^{w},
$$

where $g$ is a GUT element, and so the GUT group is broken.

Being known as holonomies, these quantities also furnish a representation of the fundamental group. So if the fundamental group is taken to be the abelian $\mathbf{Z}_{\mathbf{n}}$, then $\mathscr{W}^{n}=1$. From now on we will assume this is the case, and that our compact space has $\mathbf{Z}_{\mathbf{n}}$ as the fundamental group. Since the fundamental group is abelian, the Wilson lines will be rank preserving, and as such the GUT group can be broken to

$$
S O(10) \rightarrow S U(3) \times S U(2) \times U(1) \times U(1)
$$

Being mutually commuting elements of both the GUT group and the surviving group, Wilson lines are elements of the centre of the surviving group (as they also commute with the surviving group). Therefore, they can be taken to be generated by the generators of the surviving $U(1)$ factors. Hence, a useful way to represent a Wilson line is by analogy with the way one normally writes down group elements,

$$
\mathscr{W}=\sum_{m} \frac{1}{m !}\left(\frac{i 2 \pi}{n} \sum_{j} a_{j} Q_{j}\right)^{m},
$$

which makes the properties of the Wilson line explicit, namely $\mathscr{W}^{n}=1$.

The above representation explicitly shows that $\mathscr{W}$ are diagonal matrices whose entries are $n^{\text {th }}$ roots of unities to some power given by the charges of the $U(1)$ factors. Witten [18] realised that these phases are crucial for model building. If the geometry of the compact space admits a symmetry isomorphic to the fundamental group, i.e. $\mathbf{Z}_{\mathbf{n}}$, then the action of the discrete group and the gauge group will mix. As a result, the phases in $\mathscr{W}$ become charges of the discrete symmetry. For example, in $S U(5)$ models, $\mathscr{W}$ takes the form

$$
\mathscr{W}=\operatorname{diag}\left(\eta^{\gamma}, \eta^{\gamma}, \eta^{\gamma}, \eta^{\delta}, \eta^{\delta}\right)
$$

with $3 \gamma+2 \delta=0 \bmod n$, and if it is absorbed by a 5 containing a MSSM Higgs, the Higgs and coloured triplets will have different discrete charges, leading to a solution for the doublet-triplet splitting problem implemented in the $G_{2}$-MSSM.

The tree-level superpotential terms - those allowed by gauge symmetry and by the discrete symmetry - will have coefficients given by the action of membrane instantons. The action of the instantons is a function of the homology classes of the 3-cycles encapsulating the relevant singularities, and can be parametrised by the distances between them. For example, take a trilinear superpotential coupling between three supermultiplets $X, Y$, and $Z$, we have then

$$
y_{X Y Z} \sim \exp \left(-\operatorname{vol}_{X Y Z}\right),
$$

with the overall coefficient being a complex number with magnitude $\mathscr{O}(1)$. The bilinear terms - the $\mu$-type mass parameters - have suppressions of a GUT scale mass parameter, which can take either small or large values depending on the distance between the singularities supporting the superfields 
involved. Furthermore, one can find that the unified gauge coupling is related to the volume of the seven dimensional internal space, $V_{7}$,

$$
V_{7} \sim \frac{1}{\alpha_{G U T}^{7 / 3}}
$$

The specific values for the above quantities can only be computed if one has a full description of the geometry of the internal space. While it is true that there are no known explicit examples of compact $G_{2}$-manifolds yet, the above considerations give us a guideline for the values of these parameters.

So far the construction presented accounts for a global $\mathscr{N}=1$ SUSY field theory with a broken GUT group and a natural discrete symmetry. This symmetry can be used to prevent couplings, namely $\mu$-terms between vector-like pairs like the MSSM Higgses. On the other hand, direct searches for Higgsinos tell us that the $\mu \geq 100 \mathrm{GeV}$. The discussed framework has a natural way of generating $\mu$-terms in $M$ Theory a la Giudice-Masiero mechanism [19], to see this note that $M$ Theory compactified on $G_{2}$-manifolds has only geometric moduli, $s_{i}$, which are pared up with axions $\tau_{i}$ to form the complex scalar component of a supermultiplet. Due to due absence of fluxes, the axions enjoy an exact shift-symmetry that prevent the corresponding superfield to have a perturbative superpotential. Therefore, moduli can only couple perturbatively to chiral supermultiplets supported by singularities through Kahler potential interactions. Expanding the Kahler potential to lower order in the visible matter fields one finds for example

$$
K \supset \frac{s}{m_{p l}} H_{u} H_{d}+\text { c.c. }
$$

where $s$ denotes a generic modulus. It was studied in [20] that strong gauge dynamics in a hidden sector generate contributions to the moduli potential, creating a minimum that stabilises all the moduli. In the process, the moduli and their F-terms acquire non-vanishing vevs, spontaneously breaking SUSY and the discrete geometric symmetry. Numerical enquires [7, 8, 9, 20] shown that $\langle s\rangle \sim 0.1 m_{p l}$, with SUSY breaking non-vanishing $F$-terms vevs being $\left\langle F_{s}\right\rangle \sim \mathscr{O}(0.01) m_{3 / 2} m_{p l}$, while the gravitino mass

$$
m_{3 / 2} \sim \mathscr{O}(10 \mathrm{TeV}),
$$

as such effective $\mu$-terms can be generated from interactions of the form of Eq. 2.9 a la GiudiceMasiero

$$
\mu=\left\langle m_{3 / 2} K_{H_{u} H_{d}}-F^{k} K_{H_{u} H_{d} k}\right\rangle \simeq \mathscr{O}(1 \mathrm{TeV}) .
$$

The same mechanism can also generate trilinear terms that are otherwise forbidden by the discrete symmetry. Consider three superfields that can form a trilinear gauge invariant term which is forbidden by the discrete symmetry. Then, generically one expects that there exists a modulus or a product of different moduli such that in the Kahler potential one has

$$
K \supset \frac{s}{m_{p l}^{2}} X Y Z+\text { c.c. }
$$

and as the moduli stabilise an effective trilinear coupling is generated, and its value can be estimated to be

$$
\lambda \simeq \frac{m_{3 / 2}\langle s\rangle}{m_{p l}^{2}} \simeq \mathscr{O}\left(10^{-14}\right) .
$$


In principle the above couplings can be further suppressed if there is no single modulus with the correct discrete charge to allow for the term in the Kähler potential. Nevertheless it should be clear that generic dangerous terms and vector-like $\mu$-term masses will be generated by moduli vevs.

Apart from generating effective terms, moduli stabilisation also spontaneously break symmetry as moduli F-terms are non-vanishing. Here we skip the details of the mechanism, the outline is that under the assumption that there is a hidden sector with a strongly coupled gauge group and a mesonic field supported by appropriate conical singularities, the moduli potential is minimised and all moduli are stabilised. The gravitino mass[7]

$$
m_{3 / 2} \simeq e^{K / m_{p l}^{2}}\left|W_{h i d}\right| / m_{p l}^{2} \simeq \mathscr{O}(10 \mathrm{TeV})
$$

sets the scale for all SUSY breaking soft-parameters. Under reasonable assumptions regarding the geometry of the compact space, one finds that the soft-breaking parameters are found to be[8]

$$
\begin{aligned}
\widetilde{m}_{\bar{\alpha} \beta}^{2} & \simeq \delta_{\bar{\alpha} \beta} m_{3 / 2}^{2} \\
A_{\alpha \beta \gamma} & \simeq Y_{\alpha \beta \gamma} m^{3 / 2} \\
B \mu_{\alpha \beta} & \simeq 2 m^{3 / 2} \mu_{\alpha \beta} \\
M_{1 / 2}^{a} & \simeq 0.08 m_{3 / 2}
\end{aligned}
$$

where we notice that we have universal soft-scalar masses, and the gaugino masses are suppressed relative to the other soft-parameters. The reason for the gaugino mass suppression is that it is dominated by moduli F-terms, which have a smaller scale than the actual vevs.

Having introduced all the relevant features and generic constraints of $M$ Theory on a $G_{2}-$ manifold, we are now ready to study the most recent developments of $S O(10)$ SUSY GUT realisations.

\section{3. $S O(10)$ SUSY GUT models}

Our initial assumptions is that the compact $G_{2}$-manifold admits the appropriate conical singularities to support the MSSM spectrum, i.e. a $\mathbf{1 0}$ for the Higgses, while matter is contained in three $\mathbf{1 6}^{m}$. Furthermore, we also assume that the internal space admits an $\mathbf{Z}_{\mathbf{n}}$ discrete symmetry that is also isomorphic to the fundamental group. Under these assumptions, we can start building $S O(10)$ SUSY GUT models with a natural discrete symmetry.

As explained above, an abelian discrete Wilson line can break $S O(10)$ into

$$
S U(3) \times S U(2) \times U(1)_{Y} \times U(1)_{X},
$$

where $U(1)_{X}$ is a surviving factor that has to be broken by other mechanisms.

The Wilson line that accomplishes this is generated by the generators of $U(1)_{Y} \times U(1)_{X}$. Under a certain choice of normalisation of the Wilson line phases, one can find that such Wilson line can be absorbed into the $\mathbf{1 0}$ such that it will transform under the discrete symmetry as

$$
\mathbf{1 0} \rightarrow \eta^{\omega}\left(\eta^{-\alpha} H_{d} \oplus \eta^{\beta} \bar{D} \oplus \eta^{\alpha} H_{u} \oplus \eta^{-\beta} D\right) .
$$

where $\omega$ is the overall discrete charge, and $\alpha, \beta$ Wilson line charges, and $\eta$ the $n^{\text {th }}$ root of unity. 


\subsection{A new solution for the doublet-triplet problem}

In $S O(10)$ models, the $\mu$-term arises from the GUT coupling

$$
W \supset \mu 1010=\mu\left(H_{u} H_{d}+D \bar{D}\right)
$$

which, without any further constraint, leads to the doublet-triplet splitting problem. One could expect that the discrete charges assimilated by $\mathbf{1 0}$ from the Wilson line could be used to prevent the mass term for the coloured triplets, but since the Wilson line phases do cancel out exactly for each term one finds that disallowing a GUT-scale mass for the Higgs would be accomplished by $2 \omega \neq 0 \bmod n$, while a GUT-scale mass for the coloured triplets could be present if $2 \omega=0$ $\bmod n$. Therefore, it's not possible to employ the customary doublet-triplet splitting solution using Wilson lines like Witten shown.

One could entertain the idea of adding multiple $10 \mathrm{~s}$, in different combinations and with multiple independent Wilson lines. In that scenario one would have the superpotential mass terms

$$
W \supset \mathbf{H}_{d}^{T} \cdot \mu_{H} \cdot \mathbf{H}_{u}+\overline{\mathbf{D}}^{T} \cdot M_{D} \cdot \mathbf{D},
$$

where $\mathbf{H}_{u / d}$ are the arrays of all $H_{u / d}, \overline{\mathbf{D}}, \mathbf{D}$ the arrays for the coloured triplets, and $\mu_{H}, M_{D}$ mass parameter matrices. In order to mimic the MSSM spectrum, one would like $\mu_{H}$ to have only one vanishing eigenvalue, while $M_{D}$ has heavy eigenvalues. We can show that one cannot find a solution that accomplishes this, and as such we cannot avoid the presence of light-coloured triplets in the spectrum.

While this seems dangerous, we can use the discrete symmetry to prevent some couplings from

$$
1016{ }^{m} 16^{m},
$$

to be present in the renormalisable tree-level superpotential. Namely, we want to decouple $\bar{D}$ and $D$ from matter while retaining the Yukawa couplings

$$
y_{u}^{i j} H_{u}^{w} \mathbf{1 6}_{i}^{m} \mathbf{1 6}_{j}^{m} \equiv y_{u}^{i j} H_{u}^{w}\left(Q_{i} u_{j}^{c}+L_{i} N_{j}+i \leftrightarrow j\right) .
$$

To accomplish this, let $\mathbf{1 6}^{m}$ transform under the discrete symmetry with their overall charge $\mathbf{1 6}^{m} \rightarrow \eta^{m} \mathbf{1 6}^{m}$, i.e. without Wilson line charges, and allow for

$$
\begin{array}{ll}
H_{u}^{w} \mathbf{1 6}^{m} \mathbf{1 6}^{m}: 2 \kappa+\alpha+\omega=0 & \bmod n \\
H_{d}^{w} \mathbf{1 6}^{m} \mathbf{1 6}^{m}: 2 \kappa-\alpha+\omega=0 & \bmod n \\
D^{w} \mathbf{1 6}^{m} \mathbf{1 6}^{m}: 2 \kappa-\beta+\omega \neq 0 & \bmod n \\
\bar{D}^{w} \mathbf{1 6}^{m} \mathbf{1 6}^{m}: 2 \kappa+\beta+\omega \neq 0 & \bmod n .
\end{array}
$$

The suppression of the couplings between the coloured triplets and matter allows was discussed by Dvali in [21] and also [22, 23, 24] from a bottom-up perspective.

The presence of light coloured-triplets is not without consequences. Generically, the couplings forbidden by the discrete symmetry will be generated by moduli vev through the Kähler interactions

$$
K \supset \frac{s}{m_{p l}^{2}} D Q Q+\frac{s}{m_{p l}^{2}} D e^{c} u^{c}+\frac{s}{m_{p l}^{2}} D N d^{c}+\frac{s}{m_{p l}^{2}} \bar{D} d^{c} u^{c}+\frac{s}{m_{p l}^{2}} \bar{D} Q L
$$


that lead to the effective superpotential terms

$$
W_{e f f} \supset \lambda D Q Q+\lambda D e^{c} u^{c}+\lambda D N d^{c}+\lambda \bar{D} d^{c} u^{c}+\lambda \bar{D} Q L
$$

where we take all the couplings to be similar, $\lambda \simeq \mathscr{O}\left(10^{-14}\right)$. These terms will induce proton decay, and we can estimate the proton lifetime by

$$
\tau_{p}=\Gamma_{p}^{-1} \approx\left(\frac{\left|\lambda^{2}\right|^{2}}{16 \pi^{2}} \frac{m_{p}^{5}}{m_{D}^{4}}\right)^{-1} \approx 10^{38} \mathrm{yrs}
$$

and as such these effective terms do not pose a great threat to proton stability.

Another important consequence of these couplings is that it provides a decay channel, avoiding unrealistic relic densities and would pose a threat to BBN. The lifetime of the coloured triplets can be estimated by

$$
\tau_{D}=\Gamma_{D}^{-1} \approx\left(\lambda^{2} m_{D}\right)^{-1} \approx 0.1 \mathrm{sec},
$$

which is in agreement with BBN limits. Furthermore, being long-lived they can produce interesting collider signatures.

\subsection{The Vector-like family}

Another presented by the presence of light coloured triplets is that unification is spoiled if the spectrum is MSSM+coloured triplets, since the extra matter does not fill a full GUT irrep.

The only way we know of preserving gauge unification is to complete a GUT irrep with the coloured triplets. Such is accomplished by assuming that the internal space has appropriate singularities to support an extra vector-like family $\mathbf{1 6}_{X}, \overline{\mathbf{1 6}}_{X}$, and that $\mathbf{1 6}_{X}$ absorbs Wilson line phases such that under the discrete symmetry it transform as

$$
\mathbf{1 6}_{X} \rightarrow \eta^{x}\left(\eta^{-3 \gamma} L \oplus \eta^{3 \gamma+\delta} e^{c} \oplus \eta^{3 \gamma-\delta} N \oplus \eta^{-\gamma-\delta} u^{c} \oplus \eta^{-\gamma+\delta} d^{c} \oplus \eta^{\gamma} Q\right)
$$

while $\overline{\mathbf{1 6}}_{X}$ does not absorb any Wilson line phases, $\overline{\mathbf{1 6}}_{X} \rightarrow \eta^{\bar{x}} \overline{\mathbf{1 6}}_{X}$. As such, the condition for a complete GUT irrep requires that we integrate ou the d-type quarks, this is accomplished by allowing the mass term

$$
\overline{d c}_{X} d_{X}^{c}: x-\gamma+\delta+\bar{x}=0 \quad \bmod n,
$$

such that $\overline{d^{c}}, d_{X}^{c}$ get a GUT-scale mass if the singularities are close to each other. Unification is then preserved albeit with a higher gauge unification coupling as can be seen in Fig. 2.

The addition of the extra vector-like family is further motivated by noticing that without it there is no way of generating a Majorana mass term for the matter right-handed conjugated neutrino and therefore we would not have a see-saw mechanism to explain the light neutrino masses. On the other hand, with the vector-like family we have the term

$$
\frac{1}{m_{p l}} \overline{\mathbf{1 6}}_{X} \overline{\mathbf{1 6}}_{X} \mathbf{1 6}^{m} \mathbf{1 6}^{m}
$$

Under $S O(10)$ the Yukawa couplings are unified and the neutrinos have a Dirac mass of the same order as the up-type quarks. Consequently we can estimate the magnitude of $\left\langle\bar{N}_{X}\right\rangle$ by demanding 


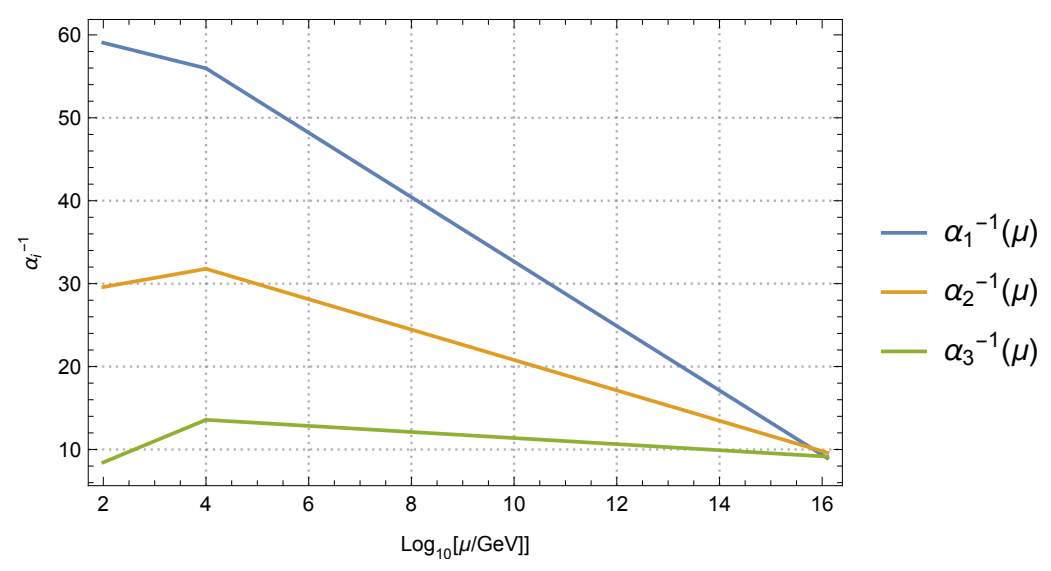

Figure 2: Gauge coupling unification with an extra vector-like family

realistic neutrino masses for the third family where $m_{v} \simeq m_{t}$, and since the physical neutrino mass is $m \simeq m_{v}^{2} / M_{m a j}$ we have $\left\langle\bar{N}_{X}\right\rangle \gtrsim 10^{16} \mathrm{GeV}$. Such high-scale vev can be accomplished for example by some sort of Kolda-Martin mechanism[25], where the system aligns in the D-flat direction, and so we will consider $\left\langle N_{X}\right\rangle=\left\langle\bar{N}_{X}\right\rangle=v_{X}$. As we will see below this high-scale vev can lead to interesting phenomena.

An immediate concern one could have is that if the generic $\mu$-terms generated by the moduli vevs coupling extra matter to regular matter

$$
\mu_{X L}^{i} \bar{L}_{X} L^{i}, \mu_{X Q}^{i} \bar{Q}_{X} Q^{i}, \mu_{X u}^{i} \bar{u}^{c} X\left(u^{c}\right)^{i}, \mu_{X e}^{i} \bar{e}^{e_{X}}\left(e^{c}\right)^{i}, \lambda^{i}\left\langle N_{X}\right\rangle D\left(d^{c}\right)^{i},
$$

can induce too much mixing. Hopefully, this is not what happens and we explain why. Consider the mass terms for the third family quark and its couplings with extra matter

$$
M=\left(\begin{array}{ccc}
0 & \mu_{1} & 0 \\
0 & \mu_{2} & 0 \\
\mu_{3} & 0 & \mu_{4}
\end{array}\right) .
$$

where we are considering only one quark and neglecting EWS masses since the mass of the top is one order of magnitude lower than the $\mu$ parameters, and the remaining off-diagonal entries are non-vanishing only to first order in $\lambda$. The Lagrangian will read schematically

$$
\mathscr{L} \supset \mu_{1} u_{3} \bar{u}_{X}+\mu_{2} u_{X} \bar{u}_{X}+\mu_{3} \overline{u^{c}} X\left(u^{c}\right)_{3}+\mu_{4}{\overline{u^{c}}}_{X} u_{X}^{c},
$$

and so one can diagonalise the system by defining the linear combinations

$$
\begin{gathered}
u_{X}^{\prime}=\frac{1}{\sqrt{\mu_{1}^{2}+\mu_{2}^{2}}}\left(\mu_{1} u_{3}+\mu_{2} u_{X}\right) \\
\left(u_{X}^{c}\right)^{\prime}=\frac{1}{\sqrt{\mu_{3}^{2}+\mu_{4}^{2}}}\left(\mu_{3}\left(u^{c}\right)_{3}+\mu_{4} u_{X}^{c}\right)
\end{gathered}
$$


which will account for two tev-scale Dirac masses

$$
\begin{aligned}
& \sqrt{\mu_{1}^{2}+\mu_{2}^{2}} u_{X}^{\prime} \bar{u}_{X} \\
& \sqrt{\mu_{3}^{2}+\mu_{4}^{2}} \bar{u}_{X}^{c}\left(u_{X}^{c}\right)^{\prime}
\end{aligned}
$$

while two Weyl fermions remain massless

$$
\begin{gathered}
t=u_{3}^{\prime}=\frac{1}{\sqrt{\mu_{1}^{2}+\mu_{2}^{2}}}\left(\mu_{2} u_{3}-\mu_{1} u_{X}\right), \\
t^{c}=\left(u_{3}^{c}\right)^{\prime}=\frac{1}{\sqrt{\mu_{3}^{2}+\mu_{4}^{2}}}\left(\mu_{4}\left(u^{c}\right)_{3}-\mu_{3} u_{X}^{c}\right),
\end{gathered}
$$

and we identify these as being the SM top-quarks. The relevance of this result is that the above linear combinations will not lead to unitary violation in the weak currents, which would lead to tree-level Flavour Changing Neutral Currents (FCNC). To see this we notice that the only up-type quark that couples differently to the weak current is the $\overline{u_{X}^{c}}$ as it is an $S U(2)$ singlet, but to leading order in $m / \mu \leq 0.1$, where $m$ is an EWS mass, this quark does not appear in the linear combination making up $t$.

\subsection{R-parity violation}

Whilst minimal $S O(10)$ models mimic an effective R-parity that $S U(5)$ models do not, the presence of extra matter can generate terms that effectively break R-parity at low energies. These R-parity violating (RPV) terms arise from the Kähler interactions

$$
K_{R P V} \supset \frac{s}{m_{p l}^{3}} \mathbf{1 6}_{X} 16^{m} 16^{m} 16^{m}+\frac{s}{m_{p l}^{2}} \mathbf{1 0}^{w} 16_{X} 16^{m} .
$$

and as both the moduli and $N_{X}, \bar{N}_{X}$ acquire vevs, the effective RPV superpotential terms

$$
W_{R P V}^{e f f} \supset \lambda \frac{v_{X}}{m_{p l}} L L e^{c}+\lambda \frac{v_{X}}{m_{p l}} L Q d^{c}+\lambda \frac{v_{X}}{m_{p l}} u^{c} d^{c} d^{c}+\lambda v_{X} L H_{u},
$$

are generated. Since the $U(1)$ breaking is taken to happen in the D-flat direction of $N_{X}, \bar{N}_{X}$ system, $v_{X} \simeq 10^{16} \mathrm{GeV}$, meaning that $\lambda v_{X} \simeq 100 \mathrm{GeV}$.

The bilinear RPV term can be rotated away by performing a small rotation $\mathscr{O}\left(v_{X} \lambda / \mu\right)$ in the $L, H_{d}$ space. Consequently, the trilinear RPV containing $L$ terms will be enhanced by contributions from the down type Yukawa couplings

$$
W_{R P V}^{e f f} \supset y_{e} \frac{v_{X}}{m_{p l}} L L e^{c}+y_{d} \frac{v_{X}}{m_{p l}} L Q d^{c}+\lambda \frac{v_{X}}{m_{p l}} u^{c} d^{c} d^{c},
$$

where we keep the same name as before for the rotated fields. Since the $u$ ud RPV term does not get enhanced, RPV does not pose a risk for proton-decay. Nonetheless the above interactions provide a decay channel for LSP. The life-time of the LSP can be roughly estimated by[26]

$$
\tau_{L S P} \simeq \frac{10^{-13} \mathrm{sec}}{\left(v_{X} / m_{p l}\right)^{2}}\left(\frac{\widetilde{m}}{10 \mathrm{TeV}}\right)^{4}\left(\frac{100 \mathrm{GeV}}{m_{L S P}}\right)^{5},
$$


and considering $v_{X} \simeq 10^{16} \mathrm{GeV}, \widetilde{m} \simeq 10^{4} \mathrm{GeV}, m_{L S P} \simeq M_{1 / 2} \simeq 100 \mathrm{GeV}$ one finds $\tau_{L S P} \simeq 10^{-9} \mathrm{sec}$. This bound is compatible with BBN constraints [27], although it is clear the the LSP cannot be the main dark matter (DM) component. Fortunately, $M$ Theory normally provides axionic DM[28, 29].

While the trilinear RPV does not pose any serious challenge to the model, the bilinear term does. Before rotation, the bilinear term represents a $\mu$-term of order $100 \mathrm{GeV}$. This is in stress with current bounds of order $1 \mathrm{GeV}$ from neutrino masses [30,31]. While this looks dangerous at first, we appeal to the discrete symmetry to argue that the Kähler term interaction generating the bilinear RPV term might be suppressed if there is no single modulus and product of two modulus that can balance the discrete charge of $N_{X} L H_{u}$, i.e. by requiring that the leading order term for this interaction in the Kähler potential takes the form

$$
K \supset \frac{s^{3}}{m_{p l}^{4}} v_{X} L H_{u},
$$

where $s$ generically refers to a product of three moduli (need not to be the same modulus to the cube). This provides an $\mathscr{O}\left(10^{-2}\right)$ suppression that would relax the see-saw requirements for $v_{X}$ and the bilinear RPV bounds.

\subsection{Example of a model}

An explicit example can be found by scanning the parameter space of the discrete charges. For the choice

$$
(N, \omega, \alpha, \beta, \kappa, x, \gamma, \delta, \bar{x})=(16,4,0,1,6,2,1,13,2),
$$

we can find a model with tree-level renormalisable superpotential

$$
W=y_{u} H_{u}\left(Q u^{c}+L N\right)+y_{d} H_{d}\left(Q d^{c}+L e^{c}\right)+M \bar{d}^{c} X d_{X}^{c},
$$

that also allows for the right-handed conjugate neutrino Majorana mass term and is anomaly free[32].

The generic runnings for masses, for $m_{3 / 2}=30 \mathrm{TeV}$, can be seen in Fig. 3, where we can see that the scalar masses are generally very heavy while the fermionic should be in reach of the LHC.

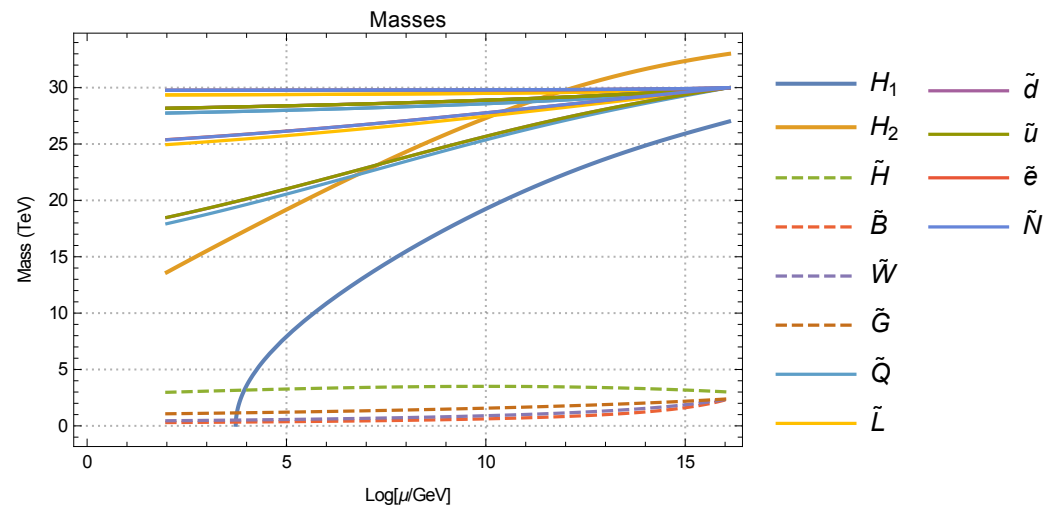

Figure 3: Runnings of some masses: full lines refer to bosons, dashed lines refer to fermions 


\section{Conclusions, prospects and future work}

We presented the generic features and predictions of $S O(10)$ SUSY GUT models arising from $M$ Theory on a $G_{2}$ manifold without fluxes. We showed that the main prediction is a TeV scale vetor-like family that is in reach of the LHC. Furthermore, the light gaugino masses at the GUT scale, in conjugation with the step gauge coupling running, provide a smoking gun for this class of models. We showed that RPV bounds can test a high-scale see-saw mechanism, providing one more way of testing and probing for this class of models. Hence, these models provide a predictive and complete framework to study in the near future with the LHC run 2 in mind.

Whilst being an interesting class of models as presented here, there is still work to be done. Namely, we need to understand better the details of the extra $U(1)$ breaking and how this affects the viability of the see-saw mechanism. These questions, regarding the interplay between the details of symmetry breaking and see-saw mechanism, are currently being study and will appear soon.

\section{Acknowledgments}

This work was funded by FCT under the grant SFRH/BD/84234/2012. The author would like to thank the collaboration with whom this work was done, Bobby S. Acharya, Krzysztof Bozek, Stephen F. King, and Chakrit Pongkitivanichkul.

\section{References}

[1] ATLAS Collaboration Collaboration, G. Aad et al., Observation of a new particle in the search for the Standard Model Higgs boson with the ATLAS detector at the LHC, Phys.Lett. B716 (2012) 1-29, [arXiv:1207.7214].

[2] CMS Collaboration Collaboration, S. Chatrchyan et al., Observation of a new boson at a mass of 125 GeV with the CMS experiment at the LHC, Phys.Lett. B716 (2012) 30-61, [arXiv: 1207.7235$].$

[3] CMS Collaboration Collaboration, Precise determination of the mass of the Higgs boson and studies of the compatibility of its couplings with the standard model, Tech. Rep. CMS-PAS-HIG-14-009, CERN, Geneva, 2014.

[4] ATLAS Collaboration Collaboration, G. Aad et al., Search for strong production of supersymmetric particles in final states with missing transverse momentum and at least three b-jets at $\sqrt{s}=8 \mathrm{TeV}$ proton-proton collisions with the ATLAS detector, JHEP 1410 (2014) 24, [arXiv: 1407.0600 ].

[5] CMS Collaboration Collaboration, S. Chatrchyan et al., Search for supersymmetry in hadronic final states with missing transverse energy using the variables $\alpha_{T}$ and b-quark multiplicity in pp collisions at $\sqrt{s}=8$ TeV, Eur.Phys.J. C73 (2013) 2568, [arXiv:1303.2985].

[6] ATLAS Collaboration Collaboration, G. Aad et al., Search for direct production of charginos and neutralinos in events with three leptons and missing transverse momentum in $\sqrt{s}=8 T e V p p$ collisions with the ATLAS detector, JHEP 1404 (2014) 169, [arXiv: 1402 . 7029 ].

[7] B. S. Acharya, K. Bobkov, G. L. Kane, P. Kumar, and J. Shao, Explaining the Electroweak Scale and Stabilizing Moduli in M Theory, Phys.Rev. D76 (2007) 126010, [hep-th/ 0701034 ].

[8] B. S. Acharya, K. Bobkov, G. L. Kane, J. Shao, and P. Kumar, The G(2)-MSSM: An M Theory motivated model of Particle Physics, Phys.Rev. D78 (2008) 065038, [arXiv: 0801.0478 ]. 
[9] B. S. Acharya, G. Kane, and P. Kumar, Compactified String Theories - Generic Predictions for Particle Physics, Int.J.Mod.Phys. A27 (2012) 1230012, [arXiv:1204.2795].

[10] S. Kachru, R. Kallosh, A. D. Linde, and S. P. Trivedi, De Sitter vacua in string theory, Phys.Rev. D68 (2003) 046005, [hep-th/0301240].

[11] V. Balasubramanian, P. Berglund, J. P. Conlon, and F. Quevedo, Systematics of moduli stabilisation in Calabi-Yau flux compactifications, JHEP 0503 (2005) 007, [hep-th/ 05020 58].

[12] B. S. Acharya, K. Bożek, M. Crispim Romão, S. F. King, and C. Pongkitivanichkul, SO(10) Grand Unification in M theory on a G2 manifold, Phys. Rev. D92 (2015), no. 5 055011, [arXiv:1502.0172].

[13] E. Witten, Symmetry Breaking Patterns in Superstring Models, Nucl.Phys. B258 (1985) 75.

[14] R. N. Mohapatra and M. Ratz, Gauged Discrete Symmetries and Proton Stability, Phys.Rev. D76 (2007) 095003, [arXiv:0707.4070].

[15] H. M. Lee, S. Raby, M. Ratz, G. G. Ross, R. Schieren, et al., A unique $Z_{4}^{R}$ symmetry for the MSSM, Phys.Lett. B694 (2011) 491-495, [arXiv: 1009.0905$].$

[16] B. S. Acharya and E. Witten, Chiral fermions from manifolds of G(2) holonomy, hep-th/0109152.

[17] B. S. Acharya and S. Gukov, $M$ theory and singularities of exceptional holonomy manifolds, Phys.Rept. 392 (2004) 121-189, [hep-th/ 0409191 ].

[18] E. Witten, Deconstruction, G(2) holonomy, and doublet triplet splitting, hep-ph/0201018.

[19] G. Giudice and A. Masiero, A Natural Solution to the mu Problem in Supergravity Theories, Phys.Lett. B206 (1988) 480-484.

[20] B. S. Acharya and K. Bobkov, Kahler Independence of the G(2)-MSSM, JHEP 1009 (2010) 001, [arXiv:0810.3285].

[21] G. Dvali, Light color triplet Higgs is compatible with proton stability: An Alternative approach to the doublet - triplet splitting problem, Phys.Lett. B372 (1996) 113-120, [hep-ph/9511237].

[22] W. Kilian and J. Reuter, Unification without doublet-triplet splitting, Phys.Lett. B642 (2006) 81-84, [hep-ph/0606277].

[23] J. Reuter, SUSY multi-step unification without doublet-triplet splitting, arXiv:0709.4202.

[24] R. Howl and S. King, Minimal E(6) Supersymmetric Standard Model, JHEP 0801 (2008) 030, [arXiv:0708.1451].

[25] C. F. Kolda and S. P. Martin, Low-energy supersymmetry with D term contributions to scalar masses, Phys. Rev. D53 (1996) 3871-3883, [hep-ph/ 9503445$].$

[26] B. S. Acharya, G. Kane, E. Kuflik, and R. Lu, Theory and Phenomenology of $\mu$ in M theory, JHEP 1105 (2011) 033, [arXiv:1102.0556].

[27] H. K. Dreiner, An Introduction to explicit R-parity violation, Adv.Ser.Direct.High Energy Phys. 21 (2010) 565-583, [hep-ph/9707435].

[28] P. Svrcek and E. Witten, Axions In String Theory, JHEP 0606 (2006) 051, [hep-th/ 0605206 ].

[29] B. S. Acharya, K. Bobkov, and P. Kumar, An M Theory Solution to the Strong CP Problem and Constraints on the Axiverse, JHEP 1011 (2010) 105, [arXiv: 1004.5138 ]. 
[30] B. S. Acharya, G. L. Kane, P. Kumar, R. Lu, and B. Zheng, R-Parity Conservation from a Top Down Perspective, JHEP 1410 (2014) 1, [arXiv: 1403.4948$].$

[31] T. Banks, Y. Grossman, E. Nardi, and Y. Nir, Supersymmetry without R-parity and without lepton number, Phys.Rev. D52 (1995) 5319-5325, [hep-ph/ 9505248 ].

[32] T. Araki, T. Kobayashi, J. Kubo, S. Ramos-Sanchez, M. Ratz, et al., (Non-)Abelian discrete anomalies, Nucl.Phys. B805 (2008) 124-147, [arXiv: 0805.0207$].$ 